\title{
Fredholm Determinants and Inverse Scattering Problems ${ }^{\star}$
}

\author{
Freeman J. Dyson
}

Institute for Advanced Study, Princeton, N.J. 08540, USA

\begin{abstract}
The Gel'fand-Levitan and Marchenko formalisms for solving the inverse scattering problem are applied together to a single set of scattering phase-shifts. The result is an identity relating two different types of Fredholm determinant. As an application of the method, an asymptotic formula of high accuracy is derived for a particular Fredholm determinant that determines the level-spacing distribution-function in the theory of random matrices.
\end{abstract}

\section{Statement of the Problem}

The inverse scattering problem was solved in the early 1950's by two different methods, one due to Gel'fand and Levitan [1] and the other to Marchenko [2]. The essential difference between the two methods is that Gel'fand and Levitan constructed a scattering potential $V(x)$ on the half-line $0<x<\infty$ by working up from the end $x=0$, while Marchenko worked down from the end $x=\infty$. In the excellent review article of Faddeev [3] and in other discussions of inverse scattering, the methods of Gel'fand-Levitan and Marchenko are presented as alternatives, each by itself being sufficient to construct the potential. Little is said about the consequences of applying both methods to the same scattering data.

The purpose of the present paper is to elucidate the relation between the Gel'fand-Levitan and Marchenko formalisms in the context of a special example. We apply the two methods to a particular scattering problem that happened to arise out of the theory of random matrices [4]. The result of applying the two methods simultaneously is an identity (4.24) linking two Fredholm determinants, one defined on the interval $[0, s]$ and the other on the interval $[s, \infty]$. The determinant on $[0, s]$ is the one that arises naturally in random-matrix theory. The determinant on $[s, \infty]$ is easily expanded into an asymptotic series in negative powers of $s$. The two inverse-scattering methods together enable us to determine the asymptotic behavior for large $s$ of the random-matrix determinant, with a

* Research sponsored by the National Science Foundation, Grant No. GP-40768X. 
precision that goes far beyond previous calculations [5]. The same combined application of Gel'fand-Levitan and Marchenko techniques could be used to derive asymptotic expansions for a wide class of Fredholm determinants.

The plan of this paper is as follows. In Section II we define the particular Fredholm determinants with which we are concerned, review their previous history, and collect the pieces of inverse-scattering theory that we shall use. In Section III we apply the Gel'fand-Levitan method (using inverse-scattering theory in reverse) to calculate the scattering phase-shifts (3.36). In Section IV we apply the Marchenko method to construct the potential corresponding to these phaseshifts. In Section $\mathrm{V}$ we assemble the results and derive asymptotic expansions (5.16), (5.17) for the potentials and the Fredholm determinants. In Section VI we check the accuracy of the expansions numerically.

\section{Historical}

In this paper we shall study the particular Fredholm determinants

$$
D_{ \pm}(s)=\operatorname{Det}\left[1-f_{ \pm}\right]_{0}^{s},
$$

where $f_{+}$and $f_{-}$are kernels defined by

$$
f_{ \pm}(x, y)=\frac{1}{\pi}\left[\frac{\sin (x-y)}{x-y} \pm \frac{\sin (x+y)}{x+y}\right],
$$

or alternatively by

$$
\begin{aligned}
& f_{+}(x, y)=(2 / \pi) \int_{0}^{1} \cos k x \cos k y d k, \\
& f_{-}(x, y)=(2 / \pi) \int_{0}^{1} \sin k x \sin k y d k .
\end{aligned}
$$

These determinants play an important role in the theory of random matrices [4]. Gaudin [6] proved that $D_{+}(s)$ is the probability for finding no eigenvalue of a random real symmetric matrix of large order in a given interval of length $(2 \mathrm{Ls} / \pi)$ within a region where the mean eigenvalue-spacing is $L$. The determinant $D_{-}(s)$ is the probability for finding at most one eigenvalue in the same interval. Gaudin [6] showed that the eigen-functions of the kernels $f_{ \pm}$are spheroidal functions, and used this fact to compute $D_{+}(s)$ numerically. Appendix A 12 of Mehta's book [4] contains numerical tables of $D_{+}$and $D_{-}$and series expansions of these functions in ascending powers of s. Appendix A 31 contains a proof (due to Gaudin) of Mehta's identity

$$
D_{+} D_{-}^{\prime \prime}+D_{+}^{\prime \prime} D_{-}=2 D_{+}^{\prime} D_{-}^{\prime},
$$

in which the primes denote differentiation with respect to $s$. The behavior of $D_{ \pm}(s)$ for large $s$ was investigated by Des Cloiseaux and Mehta [5]. They found the asymptotic formula

$$
\log D_{ \pm}(s) \sim-\frac{1}{4} s^{2} \mp \frac{1}{2} s-\frac{1}{8} \log s \pm \frac{1}{4} \log 2+B,
$$

with an error tending to zero as $s \rightarrow \infty$, the constant $B$ being undetermined. This contradicted an earlier conjecture by the present author [7]. 
The constant $B$ had already been calculated in an entirely different context by Widom [8]. Widom was concerned with Toeplitz determinants

$$
\begin{aligned}
& \Delta_{N}(\varphi)=\operatorname{Det}\left[c_{j-k}\right]_{j, k=1, \ldots, N}, \\
& c_{n}=(2 \pi)^{-1} \int_{0}^{2 \pi} \varphi(x) \exp (-i n x) d x .
\end{aligned}
$$

The classical theorem of Szegö [9] determined the asymptotic behavior of $\Delta_{N}(\varphi)$ for large $N$, in the case when $\varphi$ is a smooth and non-vanishing function. Widom extended the theorem to various singular cases, first [10] to functions $\varphi$ vanishing at a finite number of points, and later [8] to functions vanishing on an interval. In particular, for the function $\varphi_{\alpha}$ defined by

$$
\begin{aligned}
& \varphi_{\alpha}(x)=1, \quad \alpha<x<2 \pi-\alpha, \\
& \varphi_{\alpha}(x)=0, \quad x<\alpha \quad \text { and } \quad x>2 \pi-\alpha,
\end{aligned}
$$

Widom obtained the asymptotic formula

$$
\log \Delta_{N}\left(\varphi_{\alpha}\right) \sim N^{2} \log \cos \frac{1}{2} \alpha-\frac{1}{4} \log \left(N \sin \frac{1}{2} \alpha\right)+\frac{1}{12} \log 2+3 \zeta^{\prime \prime}(-1),
$$

where $\zeta^{\prime}(s)$ is the derivative of the Riemann zeta-function. The elements of the determinant in this case are

$$
c_{j-k}=\delta_{j k}-\frac{\sin (\alpha(j-k))}{\pi(j-k)} .
$$

If now we take

$$
\alpha=(2 s / N)
$$

and let $N \rightarrow \infty$ in Eq. (2.6) and (2.11), the Toeplitz determinant becomes a Fredholm determinant,

$$
\begin{aligned}
\operatorname{Lim}_{N \rightarrow \infty} \Delta_{N}\left(\varphi_{\alpha}\right) & =\operatorname{Det}\left[1-\frac{1}{2}\left(f_{+}+f_{-}\right)\right]_{-s}^{s} \\
& =D_{+}(s) D_{-}(s) .
\end{aligned}
$$

Widom's formula (2.10) becomes in the limit

$$
\log \left(D_{+}(s) D_{-}(s)\right) \sim-\frac{1}{2} s^{2}-\frac{1}{4} \log s+2 B,
$$

with

$$
B=\frac{1}{24} \log 2+\frac{3}{2} \zeta^{\prime}(-1)=-0.219250583 .
$$

Strictly speaking, Eq. (2.10) does not imply (2.14) unless it is proved that the error in (2.10) tends to zero uniformly in $\alpha$ as $\alpha N \rightarrow \infty$. The uniformity of convergence has not been proved. But Eq. (2.14) is known to be true as a consequence of Eq. (2.5), and the dubious inference from (2.10) is needed only to fix the value of $B$. The same constant $B$ appears in a calculation of a different type of Toeplitz determinant by McCoy and $\mathrm{Wu}[11]$. In fact

$$
A=\exp (2 B)
$$

is the coefficient in Eqs. (4.30), (4.31) on page 264 of McCoy and Wu. The numerical evaluation of $A$ is discussed by McCoy and Wu in their Appendix B. 
A third context in which Fredholm determinants have arisen is in the theory of the inverse scattering problem [3]. In this problem we are dealing with two potentials $V_{1}(x), V_{2}(x)$ on the half-line $0<x<\infty, V_{1}$ being supposed unknown and $V_{2}$ known. We have two corresponding families of wave-functions $u_{1}(k, x)$, $u_{2}(k, x)$, satisfying the wave-equations

$$
\left[\left(d^{2} / d x^{2}\right)+k^{2}-V_{j}(x)\right] u_{j}(k, x)=0,
$$

and generating spectral kernels $f_{1}, f_{2}$ defined by

$$
\delta(x-y)-f_{j}(x, y)=\int u_{j}(k, x) u_{j}(k, y) d k .
$$

In the Gel'fand-Levitan version [1] of inverse scattering theory, the wave-functions $u_{j}(k, x)$ are subject to boundary conditions at $x=0$, namely

$$
\begin{aligned}
& u_{1}(k, 0)=u_{2}(k, 0)=g q(k), \\
& u_{j}^{\prime}(k, 0)=h_{j} q(k),
\end{aligned}
$$

where $q(k)$ is a given function and $g, h_{1}, h_{2}$ are given coefficients.

The Fredholm determinants

$$
D_{j}(s)=\operatorname{Det}\left[1-f_{j}\right]_{0}^{s}
$$

are defined on the finite interval $[0, s]$, and satisfy the conditions

$$
\begin{aligned}
V_{1}(s)-V_{2}(s) & =2\left(d^{2} / d s^{2}\right)\left[\log \left(D_{1}(s) / D_{2}(s)\right)\right], \\
h_{1}-h_{2} & =g(d / d s)\left[\log \left(D_{1}(s) / D_{2}(s)\right)\right]_{s=0} .
\end{aligned}
$$

In the Marchenko version [2] of the theory, the wave-functions $u_{j}(k, x)$ are required to become asymptotically equal at infinity, thus

$$
u_{1}(k, x)-u_{2}(k, x) \rightarrow 0 \quad \text { as } \quad x \rightarrow \infty,
$$

uniformly in $k$. The potentials $V_{1}, V_{2}$ must approach each other closely enough so that the integral

$$
\int\left|V_{1}(s)-V_{2}(s)\right| s d s
$$

converges at infinity. The Fredholm determinants

$$
\Delta_{j}(s)=\operatorname{Det}\left[1-f_{j}\right]_{s}^{\infty}
$$

are defined on the infinite interval $[s, \infty]$, and

$$
V_{1}(s)-V_{2}(s)=2\left(d^{2} / d s^{2}\right)\left[\log \left(\Delta_{1}(s) / \Delta_{2}(s)\right)\right] .
$$

When the Gel'fand-Levitan and Marchenko formalisms are applied to the inverse scattering problem, it is customary to assume that the unknown wavefunctions $u_{1}(k, x)$ form a complete orthonormal set. Then Eq. (2.18) implies

$$
f_{1}=0, \quad D_{1}=\Delta_{1}=1,
$$

and the unknown potential $V_{1}$ is directly determined by the spectral kernel $f_{2}$ which contains only the known wave-functions $u_{2}(k, x)$. 


\section{Application of the Gel'fand-Levitan Method}

We apply the Gel'fand-Levitan formalism to the potentials

$$
V_{1}(s)=W_{ \pm}(s)=-2\left(d^{2} / d s^{2}\right) \log D_{ \pm}(s)-1,
$$

with the comparison potential

$$
V_{2}(s)=-1 \text {. }
$$

According to Eq. (2.5)

$$
W_{ \pm}(s) \sim-\frac{1}{4} s^{-2} \text { as } s \rightarrow \infty \text {. }
$$

We take $u_{1}(k, x)$ to be a complete orthonormal set of solutions of Eq. (2.17), so that Eq. (2.22) holds with

$$
D_{1}(s)=1, \quad D_{2}(s)=D_{ \pm}(s) .
$$

The wave-functions $u_{2}(k, x)$ must be cosines and sines of $\left(\left(k^{2}+1\right)^{\frac{1}{2}} x\right)$ in order to satisfy Eq. (2.17). We have to normalize these wave-functions so that

$$
f_{2}(x, y)=f_{ \pm}(x, y) \text {, }
$$

with $f_{2}$ given by Eq. (2.18) and $f_{ \pm}$by Eq. (2.3). Thus we require

$$
\int_{0}^{\infty} u_{2}(k, x) u_{2}(k, y) d k=(2 / \pi) \int_{1}^{\infty} \sin _{\sin }^{\cos } k x{ }_{\sin }^{\cos } k y d k,
$$

which is satisfied by choosing

$$
\begin{aligned}
u_{2}(k, x) & =s(k) \sin _{\cos }^{\cos }\left(\left(k^{2}+1\right)^{\frac{1}{2}} x\right), \\
s(k) & =(2 / \pi)^{\frac{1}{2}}\left[k^{2} /\left(k^{2}+1\right)\right]^{\frac{1}{4}} .
\end{aligned}
$$

We next have to determine the boundary conditions satisfied by $u_{1}(k, x)$ and $u_{2}(k, x)$ at $x=0$. In the even case, when $V_{1}=W_{+}$, Eq. (2.19) and (2.20) hold with

$$
q(k)=s(k), \quad g=1, \quad h_{2}=0 .
$$

In this case Eq. (2.23) gives

$$
h_{1}=-(d / d s)\left[\log D_{+}(s)\right]_{s=0}=f_{+}(0,0)=(2 / \pi) .
$$

Therefore the boundary conditions for $u_{1}$ are

$$
u_{1}(k, 0)=s(k), \quad u_{1}^{\prime}(k, 0)=(2 / \pi) s(k) .
$$

In the odd case, when $V_{1}=W_{-}$, Eq. (2.23) gives

$$
h_{1}=h_{2} \text {, }
$$

and the boundary conditions for $u_{1}$ are

$$
u_{1}(k, 0)=0, \quad u_{1}^{\prime}(k, 0)=\left(k^{2}+1\right)^{\frac{1}{2}} S(k) .
$$

We are now in a position to determine the behavior of $u_{1}(k, x)$ as $x \rightarrow \infty$. Since the potential $V_{1}$ decreases according to Eq. (3.3) at infinity, and the $u_{1}(k, x)$ 
are an orthonormal system, we have

$$
u_{1}(k, x) \sim(2 / \pi)^{\frac{1}{2}} \sin _{\sin }^{\cos }(k x+\eta(k)),
$$

where the phase-shift $\eta(k)$ must be calculated separately for the even and odd cases. Following Jost [12], we define $J(k, x)$ to be the solution of Eq. (2.17) with potential $V_{1}$ and the asymptotic behavior

$$
J(k, x) \sim \exp (i k x) \text { as } x \rightarrow \infty .
$$

The functions

$$
a(k)=J(k, 0), \quad b(k)=J^{\prime}(k, 0)
$$

are boundary values of functions analytic in the half-plane $(\operatorname{Im} k>0)$, with the symmetry property

$$
a(-k)=a^{*}(k), \quad b(-k)=b^{*}(k), \quad k \text { real },
$$

and the asymptotic behavior

$$
a(k) \sim 1, \quad b(k) \sim i k, \quad k \rightarrow \infty .
$$

The Wronskian

$$
J(-k, x) J^{\prime}(k, x)-J^{\prime}(-k, x) J(k, x)
$$

is independent of $x$. Equating its value at $x=0$ with its value at $x=\infty$, we find

$$
a^{*}(k) b(k)-a(k) b^{*}(k)=2 i k .
$$

The comparison of Eq. (3.14) with (3.15) implies

$$
u_{1}(k, x)=(2 / \pi)^{\frac{1}{2}} \operatorname{Im}_{\operatorname{Im}}^{\operatorname{Re}}[J(k, x) \exp (i \eta(k))] .
$$

Consider first the even case. Then the boundary conditions (3.11) together with Eq. (3.21) imply

$$
\begin{aligned}
& \operatorname{Re}[\exp (i \eta(k)) a(k)]=\left[k^{2} /\left(k^{2}+1\right)\right]^{\frac{1}{4}}, \\
& \operatorname{Re}[\exp (i \eta(k))(b(k)-(2 / \pi) a(k))]=0 .
\end{aligned}
$$

The function

$$
\varphi(k)=b(k)-(2 / \pi) a(k)
$$

is analytic in the upper half plane and satisfies the same conditions (3.17), (3.18) as $b(k)$. According to Eq. (3.23)

$$
\exp (i \eta(k))=i\left[\varphi^{*}(k) /|\varphi(k)|\right] .
$$

When we substitute Eq. (3.25) into (3.22) and make use of Eq. (3.20), we obtain

$$
|\varphi(k)|=\left(k^{2}\left(k^{2}+1\right)\right)^{\frac{1}{4}} .
$$


The only analytic function satisfying Eq. (3.17), (3.18) and (3.26) is

$$
\varphi(k)=[-k(k+i)]^{\frac{1}{2}} .
$$

Putting Eq. (3.27) back into (3.25), we find

$$
\exp (i \eta(k))=[(k-i) /(k+i)]^{\frac{1}{4}},
$$

and so the phase-shift is given by

$$
k \tan 2 \eta(k)=-1 \text {. }
$$

In the odd case, the boundary conditions (3.13) imply

$$
\begin{aligned}
& \operatorname{Im}[\exp (i \eta(k)) a(k)]=0, \\
& \operatorname{Im}[\exp (i \eta(k)) b(k)]=\left[k^{2}\left(k^{2}+1\right)\right]^{\frac{1}{4}} .
\end{aligned}
$$

Eq. (3.30) implies

$$
\exp (i \eta(k))=\left[a^{*}(k) /|a(k)|\right],
$$

and this with Eq. (3.31) and (3.20) gives

$$
|a(k)|=\left[k^{2} /\left(k^{2}+1\right)\right]^{\frac{1}{4}} .
$$

The analytic function $a(k)$ is then

$$
a(k)=[k /(k+i)]^{\frac{1}{2}},
$$

and Eq. (3.32) gives

$$
k \tan 2 \eta(k)=+1 \text {. }
$$

Both cases are included in the formula

$$
\eta(k)=\mp \frac{1}{2} \arctan \left(k^{-1}\right) .
$$

The potentials $W_{ \pm}(s)$ are uniquely determined [13] by the property that they give scattering states (3.14) with the phase-shifts (3.36), and no bound states.

It is a curious fact [14] that the identity (2.4) can be written in the form

$$
\left.\left[d^{2} / d s^{2}\right)-1-W_{-}(s)\right] u_{B}(s)=0,
$$

with

$$
u_{B}(s)=\left(D_{+}(s) / D_{-}(s)\right) \sim 2^{\frac{1}{2}} e^{-s} \quad \text { as } \quad s \rightarrow \infty .
$$

Thus $u_{B}(s)$ is an acceptable bound-state wave-function with energy $(-1)$ in the potential $W_{-}(s)$. However $u_{B}$ does not satisfy the boundary condition (3.13) for the odd case and is therefore irrelevant to the determination of $W_{-}$. Another alternative form of Eq. (2.4) is

$$
\left[\left(d^{2} / d s^{2}\right)-1-W_{+}(s)\right]\left(u_{B}(s)\right)^{-1}=0 .
$$

The wave-function $\left(u_{B}\right)^{-1}$ satisfies the correct boundary condition (3.11) for the even case at $s=0$, but fails to converge at infinity, and is therefore also irrelevant to the determination of $W_{+}$. 


\section{Application of the Marchenko Method}

We apply the Marchenko formalism to the potentials $V_{1}(s)$ defined by Eq. (3.1). What should we choose for the comparison potential $V_{2}(s)$ ? It is possible to carry through the analysis with $V_{2}(s)=0$. The calculations are then formally simple, but the integral (2.25) diverges in view of Eq. (3.3), and the results obtained are poorly convergent and of doubtful utility. The next most simple choice would be

$$
V_{2}(s)=-\frac{1}{4} s^{-2}
$$

This makes the integral (2.25) converge. The wave-functions $u_{2}(k, x)$ are now Bessel functions of the quantity $(k x)$, and the phase-shift produced by the potential $V_{2}(s)$ is $\left(\mp \frac{1}{4} \pi\right)$ independent of $k$. The fact that this phase-shift agrees with the phase-shift (3.36) at $k=0$ reflects the fact that $V_{1}$ and $V_{2}$ have the same behavior at infinity. But the same argument carried one step further suggests a far better choice for $V_{2}$. The phase-shift (3.36) behaves like

$$
\eta(k) \sim \mp\left(\frac{1}{4} \pi-\frac{1}{2} k\right),
$$

with an error of order $k^{3}$ for small $k$. A Bessel function of the quantity $\left(k\left(x \pm \frac{1}{2}\right)\right)$ gives the phase-shift (4.2) for all $k$. We therefore choose for $V_{2}$ the potential

$$
V_{2}(s)=-\frac{1}{4}\left(s \pm \frac{1}{2}\right)^{-2}
$$

with the expectation that this will make the difference $\left(V_{1}-V_{2}\right)$ decrease much more rapidly as $s \rightarrow \infty$. The results of the calculation justify our expectation. The singularity of $V_{2}(s)$ at $s=\frac{1}{2}$ (in the odd case) means that the analysis is valid only for $s>\frac{1}{2}$. This is not a serious limitation, since our purpose is to study the behavior of the potentials for large $s$.

The wave-functions $u_{2}(k, x)$ are solutions of the equation

$$
\left[\left(d^{2} / d x^{2}\right)+k^{2}+\frac{1}{4}\left(x \pm \frac{1}{2}\right)^{-2}\right] u_{2}(k, x)=0,
$$

with asymptotic behavior determined by Eq. (2.24). It is convenient to use the notations

$$
\begin{array}{ll}
j_{0}(z)=z^{\frac{1}{2}} J_{0}(z), & y_{0}(z)=z^{\frac{1}{2}} Y_{0}(z), \\
h_{0}^{1}(z)=z^{\frac{1}{2}} H_{0}^{1}(z), & k_{0}(z)=z^{\frac{1}{2}} K_{0}(z),
\end{array}
$$

for the Bessel functions. We take for the wave-functions $u_{1}(k, x)$ the same complete orthonormal set that we studied in Section III, with asymptotic behavior given by Eq. (3.14) and (3.36). Then Eq. (2.24) implies

$$
u_{2}(k, x)=\alpha(k) j_{0}\left(k\left(x \pm \frac{1}{2}\right)\right) \pm \beta(k) y_{0}\left(k\left(x \pm \frac{1}{2}\right)\right),
$$

with

$$
\begin{aligned}
& \alpha(k)=\cos \left(\frac{1}{2} \theta(k)\right), \quad \beta(k)=\sin \left(\frac{1}{2} \theta(k)\right), \\
& \theta(k)=(k-\arctan k) .
\end{aligned}
$$


The Marchenko formula (2.27) becomes

$$
\begin{aligned}
V_{1}(s) & =W_{ \pm}(s)=-\frac{1}{4}\left(s \pm \frac{1}{2}\right)^{-2}-2\left(d^{2} / d s^{2}\right) \log \Delta_{ \pm}(s), \\
\Delta_{ \pm}(s) & =\operatorname{Det}\left[1-F_{ \pm}\right]_{s}^{\infty},
\end{aligned}
$$

with the kernels $F_{ \pm}$defined by

$$
F_{ \pm}(x, y)=\delta(x-y)-\int_{0}^{\infty} u_{2}(k, x) u_{2}(k, y) d k .
$$

Using the completeness relation

$$
\delta(x-y)=\int_{0}^{\infty} j_{0}\left(k\left(x \pm \frac{1}{2}\right)\right) j_{0}\left(k\left(y \pm \frac{1}{2}\right)\right) d k,
$$

we bring Eq. (4.12) to the form

$$
\begin{aligned}
& F_{ \pm}(x, y)=\frac{1}{2} \int_{0}^{\infty} \operatorname{Re}[(1-\exp (\mp i \theta(k))) \\
& \left.h_{0}^{1}\left(k\left(x \pm \frac{1}{2}\right)\right) h_{0}^{1}\left(k\left(y \pm \frac{1}{2}\right)\right)\right] d k=\frac{1}{4}\left[\int_{0}^{\infty}-\int_{-\infty}^{0}\right](1-\exp (\mp i \theta(k))) \\
& h_{0}^{1}\left(k\left(x \pm \frac{1}{2}\right)\right) h_{0}^{1}\left(k\left(y \pm \frac{1}{2}\right)\right) d k .
\end{aligned}
$$

The function

$$
\exp (\mp i \theta(k))=\exp (\mp i k)(1 \pm i k)\left(k^{2}+1\right)^{-\frac{1}{2}}
$$

is analytic in the upper half plane with a cut from $(+i)$ to $(+i \infty)$. In the even case, the exponential growth of Eq. (4.15) is compensated by the exponential decrease of the Hankel functions in Eq. (4.14) for all positive $x$ and $y$. In the odd case, Eq. (4.15) decreases exponentially in the upper half-plane, but we must require $x, y>\frac{1}{2}$ so that the term in Eq. (4.14) not involving (4.15) has exponential decrease. With this proviso, we may move the path of integration in both parts of Eq. (4.14) to the positive imaginary axis by writing $k=i z$. The terms involving Eq. (4.15) cancel along the cut, and we are left with

$$
\begin{aligned}
F_{ \pm}(x, y) & =\left(2 / \pi^{2}\right)\left[\int_{0}^{\infty} d z-\int_{0}^{1} \exp ( \pm \varphi(z)) d z\right] k_{0}\left(z\left(x \pm \frac{1}{2}\right)\right) k_{0}\left(z\left(y \pm \frac{1}{2}\right)\right) \\
\phi(z) & =z-\operatorname{arctanh} z .
\end{aligned}
$$

Since

$$
k_{0}(u)<(\pi / 2)^{\frac{1}{2}} e^{-u}
$$

it follows from Eq. (4.16) that

$$
\left|F_{ \pm}(x, y)\right|<\pi^{-1}(x+y \pm 1)^{-2}<\left[4 \pi\left(x \pm \frac{1}{2}\right)\left(y \pm \frac{1}{2}\right)\right]^{-}
$$

The crude estimate (4.19) shows that the largest eigenvalue of the kernel $F_{ \pm}$on the interval $[s, \infty]$ is smaller than

$$
\left[4 \pi\left(s \pm \frac{1}{2}\right)\right]^{-1} \text {. }
$$

The series expansion

$$
\log \Delta_{ \pm}(s)=\operatorname{Tr}\left[\log \left(1-F_{ \pm}\right)\right]_{s}^{\infty}=-\sum_{1}^{\infty} n^{-1} \operatorname{Tr}\left[\left(F_{ \pm}\right)^{n}\right]_{s}^{\infty}
$$


converges absolutely for all positive $s$ in the even case, and at least for

$$
s>\frac{1}{2}+(4 \pi)^{-1}
$$

in the odd case. The formula (4.10) with (4.16) and (4.21) provides a practical method for computing the potentials $W_{ \pm}(s)$, either by using the series expansion or by finding numerically the eigenvalues of the kernels $F_{ \pm}$.

The relations (3.1) and (4.10), connecting $W_{ \pm}(s)$ with $D_{ \pm}(s)$ and $\Delta_{ \pm}(s)$, have the consequence that the quantity

$$
\log D_{ \pm}(s)+\frac{1}{4} s^{2}+\frac{1}{8} \log \left|s \pm \frac{1}{2}\right|-\log \Delta_{ \pm}(s)
$$

is a linear function of $s$. But we know that as $s \rightarrow \infty$ the behavior of $\log D_{ \pm}(s)$ is governed by Eq. (2.5), while $\log \Delta_{ \pm}(s)$ tends to zero. The asymptotic formula (2.5) can therefore be replaced by the identity

$$
\log D_{ \pm}(s)=-\frac{1}{4} s^{2} \mp \frac{1}{2} s-\frac{1}{8} \log \left|s \pm \frac{1}{2}\right| \pm \frac{1}{4} \log 2+B+\log \Delta_{ \pm}(s) .
$$

This identity establishes the desired connection between the Fredholm determinants defined on $[0, s]$ and those defined on $[s, \infty]$.

\section{Asymptotic Expansions}

We wish to obtain the asymptotic expansion of $\log \Delta_{ \pm}(s)$ in negative powers of $\left(s \pm \frac{1}{2}\right)$. We can then use Eq. (4.24) to obtain the extension to negative powers of the asymptotic formula (2.5) for $\log D_{ \pm}(s)$. The expansion of $\log \Delta_{ \pm}(s)$ is derived from Eq. (4.21), the $n^{\prime}$ th term of the sum giving contributions of order $s^{-3 n}$ and higher. We expand the integrand of Eq. (4.16) in powers of $z$, so that

$$
F_{ \pm}(x, y)= \pm \frac{1}{3} F_{3} \pm \frac{1}{5} F_{5}-\frac{1}{18} F_{6} \pm \frac{1}{7} F_{7}+\ldots,
$$

with the term

$$
F_{m}=\left(2 / \pi^{2}\right) \int_{0}^{\infty} z^{m} k_{0}\left(z\left(x \pm \frac{1}{2}\right)\right) k_{0}\left(z\left(y \pm \frac{1}{2}\right)\right) d z
$$

homogeneous of degree $(-m-1)$ in $\left(x \pm \frac{1}{2}\right)$ and $\left(y \pm \frac{1}{2}\right)$. The error involved in extending the integral from 1 to $\infty$ is of order $e^{-s}$ and is negligible in an asymptotic expansion. When the trace of $\left(F_{ \pm}\right)^{n}$ is calculated, we obtain terms proportional to $\left(s \pm \frac{1}{2}\right)^{-N}$ by forming products

$$
F_{m_{1}} F_{m_{2}} \ldots F_{m_{n}}
$$

with

$$
m_{1}+m_{2}+\ldots+m_{n}=N \text {. }
$$

In Eq. (5.1) the odd terms carry the \pm sign while the even terms do not. Equation (5.4) implies that terms with even $N$ appear with the same coefficients in $\log \Delta_{+}$and $\log \Delta_{-}$, while terms with odd $N$ have equal and opposite coefficients. Therefore

$$
\log \Delta_{ \pm}(s)=\Sigma( \pm 1)^{m} a_{m}\left(s \pm \frac{1}{2}\right)^{-m} .
$$


Moreover

$$
a_{1}=a_{2}=a_{4}=0,
$$

because no term of these orders can appear in any trace of $\left[F_{ \pm}\right]^{n}$ according to Eq. (5.1). The formal relation

$$
\log \Delta_{ \pm}(-s)=\log \Delta_{\mp}(s)
$$

holds for the asymptotic expansions (5.5), but cannot hold as an identity. On the contrary, if $\Delta_{ \pm}(-s)$ is defined by Eq. (4.24), using the convention

$$
D_{ \pm}(-s)=\operatorname{Det}\left[1+f_{ \pm}\right]_{0}^{s}
$$

which is the correct analytic continuation of Eq. (2.1), then Eq. (5.7) is definitely false. There is nothing paradoxical in this failure of Eq. (5.7), since the expansion (5.5) is not convergent.

The coefficients $a_{m}$ in Eq. (5.5) can all be computed as traces of products of kernels (5.2). But we can find the first two non-vanishing coefficients $a_{3}$ and $a_{5}$ without calculating any integrals, by using the identity (2.4). It is convenient to work with the derivatives

$$
L_{ \pm}(s)=(d / d s) \log D_{ \pm}(s)=-\frac{1}{2} s \mp \frac{1}{2}-\frac{1}{8}\left(s \pm \frac{1}{2}\right)^{-1}+(d / d s) \log \Delta_{ \pm}(s) ，
$$

and their sum and difference

$$
U=L_{+}+L_{-}, \quad V=L_{+}-L_{-} .
$$

The identity (2.4) then becomes

$$
U^{\prime}+V^{2}=0 \text {. }
$$

Equations (5.9) and (5.5) give to order $s^{-6}$

$$
\begin{aligned}
& U=-s-\frac{1}{4} s\left(s^{2}-\frac{1}{4}\right)^{-1}+12 a_{3} s^{-5} \\
& V=-1+\frac{1}{8}\left(s^{2}-\frac{1}{4}\right)^{-1}-6 a_{3} s^{-4}-\left(15 a_{3}+10 a_{5}\right) s^{-6} .
\end{aligned}
$$

When we substitute Eq. (5.12) into (5.11), we find that all terms vanish to order $s^{-6}$ provided that

$$
\begin{aligned}
& a_{3}=-(3 / 256), \\
& a_{5}=-(45 / 2048) .
\end{aligned}
$$

The vanishing of Eq. (5.11) provides a check of the consistency of our procedures. Unfortunately it is not possible to determine the coefficients beyond $a_{5}$ in this way, because Eq. (5.11) gives only one equation for each pair of unknowns $\left(a_{2 m}, a_{2 m+1}\right)$.

Putting together Eq. (5.5), (5.13), (5.14) we have the asymptotic expansion of $\log \Delta_{ \pm}(s)$ in powers of $s^{-1}$,

$$
\log \Delta_{ \pm}(s)=\mp \frac{3}{256} s^{-3}+\frac{9}{512} s^{-4} \mp \frac{81}{2048} s^{-5}+\ldots .
$$


Table 1

\begin{tabular}{|c|c|c|c|c|c|c|}
\hline \multirow[t]{2}{*}{$s$} & \multicolumn{3}{|l|}{$D_{+}(s)$} & \multicolumn{3}{|l|}{$D_{-}(s)$} \\
\hline & $M$ & $F_{5}$ & $T$ & $M$ & $F_{5}$ & $T$ \\
\hline 0 & 1 & 0.469449 & 1.041518 & 1 & - & - \\
\hline 1 & 0.426889 & 0.426129 & 0.428850 & 0.93720 & 2.09799 & 0.94564 \\
\hline 2 & 0.115153 & 0.115155 & 0.115267 & 0.64362 & 0.64607 & 0.64197 \\
\hline 3 & 0.019199 & 0.019199 & 0.019205 & 0.28475 & 0.28476 & 0.28449 \\
\hline 4 & 0.001961 & 0.001961 & 0.001962 & 0.07817 & 0.07817 & 0.07815 \\
\hline 5 & 0.0001222 & 0.0001223 & 0.0001223 & 0.01316 & 0.01316 & 0.01316 \\
\hline
\end{tabular}

Numerical values of the Fredholm determinants (2.1). $M$, exact from Mehta [4]; $F_{5}$, from Eq. (5.17); $T$, from Eq. (6.2).

The potentials which give rise to the phase-shifts (3.36) have the expansion

$$
W_{ \pm}(s)=-\frac{1}{4} s^{-2} \pm \frac{1}{4} s^{-3}-\frac{3}{16} s^{-4} \pm \frac{13}{32} s^{-5}-\frac{25}{32} s^{-6} \pm \frac{1239}{512} s^{-7}-\ldots .
$$

Finally, the Fredholm determinants (2.1) with which we began have the expansions

$$
\begin{aligned}
\log D_{ \pm}(s)= & -\frac{1}{4} s^{2} \mp \frac{1}{2} s-\frac{1}{8} \log \left|s \pm \frac{1}{2}\right| \\
& \pm \frac{1}{4} \log 2+B \mp \frac{3}{256}\left(s \pm \frac{1}{2}\right)^{-3} \\
& \mp \frac{45}{2048}\left(s \pm \frac{1}{2}\right)^{-5}-\ldots
\end{aligned}
$$

\section{Numerical Values}

Table 1 gives a sample of numerical values of $D_{ \pm}(s)$ to check the accuracy of the asymptotic expansions. The column headed $M$ give exact values taken from Appendix A 12 of Mehta [4]. The correspondence between Mehta's notations and ours is as follows:

$$
t=(2 / \pi) s, \quad E(t)=D_{+}(s), \quad E^{\prime}(t)=D_{-}(s) .
$$

The columns headed $F_{5}$ are calculated from the asymptotic formula (5.17) omitting terms beyond $\left(s \pm \frac{1}{2}\right)^{-5}$. The columns $T$ are calculated from the truncated formula

$$
D_{ \pm}(s)=2^{(1 / 24) \pm(1 / 4)}\left(s \pm \frac{1}{2}\right)^{-(1 / 8)} \exp \left(-\frac{1}{4} s^{2} \mp \frac{1}{2} s+\frac{3}{2} \zeta^{\prime}(-1)\right)
$$

obtained by omitting from Eq. (5.17) all negative powers of $\left(s \pm \frac{1}{2}\right)$. The table shows that Eq. (5.17) is very accurate for $D_{+}(s)$ with $s \geqq 2$ and for $D_{-}(s)$ with $s \geqq 3$, while even the truncated Eq. (6.2) is good to $1 \%$ over the range $s \geqq 1$ for both $D_{+}$and $D_{-}$. There is a marked improvement in accuracy in going from Eq. (2.5) to $(6.2)$.

Acknowledgement. I am indebted to Madan-Lal Mehta and Harold Widom for many helpful conversations and letters. Their independent disproofs of my conjectured asymptotic formula [7] for $D_{ \pm}(s)$ were the driving force that compelled me finally to do the job right. 
Note Added in Proof. Additional coefficients beyond Eq. (5.14) are

$$
\begin{aligned}
& a_{6}=3^{2} \cdot 7 \cdot 2^{-12}, \\
& a_{7}=-3^{2} \cdot 5^{3} \cdot 7 \cdot 2^{-16}, \\
& a_{8}=3^{2} \cdot 5^{3} \cdot 23 \cdot 2^{-17} .
\end{aligned}
$$

The identity (2.4) was independently discovered by H. Cornille, J. Math. Phys. 11, 61 (1970).

\section{References}

1. Gel'fand, I. M., Levitan, B. M.: Izv. Akad. Nauk SSSR, Ser. Mat. 15, 309-360 (1951); English translation in Am. Math. Soc. Translations (2) 1, 253-304 (1955)

2. Marchenko, V.A.: Dokl. Akad. Nauk SSSR 72, 457-460 (1950); 104, 695-698 (1955)

3. Faddeev, L. D.: Usp. Mat. Nauk 14, Part 4, 57-119 (1959); English translation in J. Math. Phys. 4, $72-104(1963)$

4. Mehta, M.L.: Random Matrices and the Statistical Theory of Energy Levels, chapters 5 and 6. New York: Academic Press 1967

5. Des Cloiseaux, J., Mehta, M. L. : J. Math. Phys. 14, 1648-1650 (1973)

6. Gaudin, M.: Nucl. Phys. 25, 447-458 (1961)

7. Dyson, F. J.: J. Math. Phys. 3, 157-165 (1962)

8. Widom, H. : Indiana Univ. Math. J. 21, 277-283 (1971)

9. Grenander, U., Szegö, G.: Toeplitz Forms and their Applications, p. 76. Berkeley: University of California Press 1958

10. Widom, H. : Am. J. Math. 95, 333-383 (1973)

11. McCoy,B.M., Wu,T.T.: The Two-dimensional Ising Model. Cambridge, Mass.: Harvard University Press 1973

12. Jost, R.: Helv. Phys. Acta 20, 256-266 (1947)

13. Levinson, N.: Kgl. Dansk. Vidensk. Selsk. Mat.-fys. Medd. 25, No. 9, 1-29 (1949)

14. Gaudin, M.: Letter to M.L. Mehta dated May 23, 1967. I am indebted to M. L. Mehta for a copy of this letter

Communicated by K. Hepp

Received November 6, 1975 
